\title{
CORRESPONDENCE
}

\section{ERRATA TO THE REPORTING OF THE 1995 WCCC}

Ernst A. Heinz ${ }^{1}$ has been kind enough to draw our attention to a regrettable omission in our publishing the first round of results of the $8^{\text {th }}$ World Computer-Chess Championship (ICCA Journal, Vol. 18, No. 2, p. 103). On p. 103, one game ZEUS 3.0 - NIGHTMARE was inadvertently duplicated, whereas the game DARK THOUGHT - PANDIX in the same round was not published at all. We hasten to supply the information missed by our readers.

\section{DARK THOUGHT - PANDIX (NicKey RL 25.4)}

1. 4 e5 2. Nf3 Nc6 3. Bb5 a6 4. Ba4 Nf6 5. 0-0 Be7 6. Re1 b5 7. Bb3 0-0 8. c3 d6 9. h3 Nb8 10. d4 Nbd7 11. Nbd2 Bb7 12. Bc2 c5 13. Nf1 Re8 14. Ng3 Bf8 15. Bg5 h6 16. Bxf6 Nxf6 17. d5 Bc8 18. Qe2 Be7 19. Nd2 Qc7 20. Ndf1 Bd7 21. Ne3 g6 22. c4 Bf8 23. Bd3 Reb8 24. Racl b4 25. h4 b3 26. Ra1 bxa2 27. Rxa2 Rb3 28. Bc2 Rb6 29. Real Qb7 30. b3 Bg7 31. h5 Qc7 32. Ra5 Ra7 33. hxg6 fxg6 34. Qe1 Ra8 35 . R1a2 Qb7 36. Qd1 Rf8 37. Qa1 Bc8 38. Bd1 Qd7 39. Qc3 Bb7 40. Nc2 h5 41. Qe3 h4 42. Nf1 Kh7 43. Ne1 Bh6 44. Qd3 Kg8 45. Ne3 Rf7 46. Be2 Bf4 47. Nf3 h3 48. Bf1 hxg2 49. Nxg2 Bh6 50. Nh2 Rh7 51. Ral Bg5 52. Ne3 Bxe3 53. fxe3 Rh4 54. Bg2 Kg7 55. R5a3 Rb4 56. Rf1 Bc8 57. Ra5 Qc7 58. Rfal Qb6 59. R5a3 Qb8 60. Qc2 Bb7 61. Bf3 Qc8 62. Qd3 Nh7 63. Bg2 Ng5 64. R3a2 Qf8 65. Rd2 Nh3+ 66. Bxh3 Rxh3 67. Rg2 Qd8 68. Raa2 Qb6 69. Qf1 Rxh2 70. Rxg6+ Kxg6 71. Rxh2 Qc7 72. Qf5+ Kg7 73. Rg2+ Kh8 74. Qf6+ Kh7 75. Qh4\# 1-0

Also due to Mr. Heinz's diligence we now see that the final standings on p. 102 of the same issue might well have been published in a better fashion, strictly following the SOP (sum of opponents' points) criterion, by which the ranking would have been more immediately visible. In order to perpetuate the true record, a corrected version of the final standings table follows as an erratum to the one shown in the previous issue.

Final Standings

\begin{tabular}{|c|c|c|c|c|c|c|c|c|c|}
\hline \multirow[b]{2}{*}{ pos. } & \multirow[b]{2}{*}{ program } & \multirow[b]{2}{*}{ country } & \multicolumn{5}{|c|}{ rounds } & \multicolumn{2}{|r|}{ points } \\
\hline & & & 1 & 2 & 3 & 4 & 5 & total & SOP \\
\hline 1. & FRITZ & Netherlands/Germany & 11b- & $15 w+$ & $18 b+$ & $14 \mathrm{w}+$ & $3 b+$ & 4.0 & $12^{2}$ \\
\hline 2. & *SOCRATES & USA & $3 b-$ & $14 w+$ & $23 b+$ & $9 w+$ & $8 b+$ & 4.0 & 12.5 \\
\hline 3. & DEEP BLUE & USA & $2 w+$ & $8 b+$ & $12 w+$ & $6 b=$ & $2 w-$ & 3.5 & 16.5 \\
\hline 4. & FRENCHESS & France & $6 w=$ & $7 \mathrm{~b}-$ & $17 \mathrm{~b}+$ & $12 w+$ & $14 \mathrm{~b}+$ & 3.5 & 12.5 \\
\hline 5. & JUNIOR & Israel & $8 b-$ & $16 w=$ & $24 b+$ & $19 w+$ & $11 b+$ & 3.5 & 10.5 \\
\hline 6. & WCHESS & USA & $4 b=$ & $10 w+$ & $11 w=$ & $3 w=$ & $7 b=$ & 3.0 & 15.5 \\
\hline 7. & Chess Genius & UK & $10 \mathrm{~b}=$ & $4 w+$ & $9 b=$ & $11 w=$ & $6 w=$ & 3.0 & $15(46)$ \\
\hline 8. & HITECH & USA & $5 w+$ & $3 w-$ & $15 b+$ & $16 b+$ & $2 w-$ & 3.0 & $15(45.5)$ \\
\hline 9. & REBEL & Netherlands & $13 w+$ & $11 b=$ & $7 w=$ & $2 b-$ & $20 w+$ & 3.0 & 14 \\
\hline 10. & ZUGZWANG & Germany & $7 w=$ & $6 b-$ & $22 b=$ & $15 w+$ & $17 w+$ & 3.0 & 11.5 \\
\hline 11. & SCHACH 3.0 & Germany/Netherlands & $1 w+$ & $9 w=$ & $6 b=$ & $7 b=$ & $5 w-$ & 2.5 & 16.5 \\
\hline 12. & CHEIRON & Germany & $22 b+$ & $24 w+$ & $3 b-$ & $4 b-$ & $13 w=$ & 2.5 & 12 \\
\hline 13. & VIRTUA Chess & France & $9 b-$ & $17 w+$ & $14 b-$ & $23 w+$ & $12 b=$ & 2.5 & 10.5 \\
\hline 14. & DARK THOUGHT & Germany & $19 w+$ & $2 b-$ & $13 w+$ & $1 b-$ & $4 w-$ & 2.0 & 16 \\
\hline 15. & FERRET & USA & $20 w+$ & 1b- & $8 w-$ & 10b- & $21 w+$ & 2.0 & $13.5(42.5)$ \\
\hline 16. & Sos & Germany & $18 b+$ & $5 b=$ & $19 w=$ & $8 w-$ & $9 b-$ & 2.0 & 13.5 (39) \\
\hline 17. & ZEUS 3.0 & Spain & $21 w+$ & $13 b-$ & $4 w-$ & $18 w+$ & $10 \mathrm{~b}-$ & 2.0 & 12.5 \\
\hline 18. & PHOENIX & Canada & $16 w-$ & $20 \mathrm{~b}+$ & $1 w-$ & $17 \mathrm{~b}-$ & $23 w+$ & 2.0 & $11(35)$ \\
\hline 19. & PANDIX & Hungary & $14 \mathrm{~b}-$ & $21 w+$ & $16 b=$ & $5 b-$ & $20 w=$ & 2.0 & $11(31)$ \\
\hline 20. & ULYSSES & Germany & $15 b-$ & $18 w-$ & $21 b=$ & $24 w+$ & $19 b=$ & 2.0 & 8.5 \\
\hline 21 . & NIGHTMARE & Germany & $17 \mathrm{~b}-$ & $19 b-$ & $20 w=$ & $22 w+$ & $15 b-$ & 1.5 & 9.5 \\
\hline 22. & GANDALF & Denmark & $12 w-$ & $23 b=$ & $10 w=$ & $21 b-$ & $24 w=$ & 1.5 & 9 \\
\hline 23. & LCHESS & Netherlands & $24 b=$ & $22 w=$ & $2 w-$ & $13 b-$ & $18 \mathrm{~b}-$ & 1.0 & 11 \\
\hline 24. & WOODPUSHER & UK & $23 w=$ & $12 b-$ & $5 w-$ & $20 b-$ & $22 b=$ & 1.0 & 10.5 \\
\hline
\end{tabular}

\footnotetext{
1 Am Fasanengarten 5, Postfach 6980, D-76128 Karlsruhe, Germany. Email: heinze@ira.uka.de.
}

2 This ranking is due to the result of the playoff game *SOCRATES - FRITZ 0-1. 